\title{
Orthogonal Space-Time Block Codes With Sphere Packing
}

\author{
Weifeng Su, Member, IEEE, Zoltan Safar, Member, IEEE, and K. J. Ray Liu, Fellow, IEEE
}

\begin{abstract}
Orthogonal designs have received considerable attention in the development of efficient modulation and coding methods for future multi-antenna wireless communication systems due to their special properties. In this paper, we propose a class of space-time block codes constructed by combining orthogonal designs with sphere packing for an arbitrary number of transmit antennas. The structure of the orthogonal designs is exploited to guarantee full diversity, and sphere packing is used to improve the coding advantage. Space-time block code construction from block-orthogonal designs is also considered: the full-diversity property is ensured by rotating the sphere packing underlying the code, and the optimal rotation angle is determined for a class of sphere packing. Code design examples are provided for two and four transmit antennas and various transmission rates. The simulation results show that by jointly designing the symbols in the orthogonal designs, the performance of the block codes can be significantly increased.
\end{abstract}

Index Terms-Diversity product, multiple antennas, multipleinput multiple-output (MIMO) systems, orthogonal designs, spacetime block codes (STBCs), sphere packing.

\section{INTRODUCTION}

B $\mathrm{Y}$ employing multiple transmit and receive antennas and developing appropriate space-time (ST) coding and modulation, multiple-input multiple-output (MIMO) systems can significantly increase data rates in wireless communications. The performance criteria for MIMO ST coding were first derived in [1] and [2], characterizing two quantities: the diversity advantage, which describes the asymptotic error rate decrease as a function of the signal-to-noise ratio (SNR), and the coding advantage, which determines the vertical shift of the error performance curve. Since then, a large number of ST codes have been proposed, for example, [3]-[23].

Orthogonal designs have received considerable attention in designing space-time block codes (STBCs) for MIMO communication systems. The theory of orthogonal designs, which focuses on the construction of square matrices from real or complex variables in such a way that their columns are orthogonal to each other, has a long history in mathematics [24]. The first

Manuscript received April 11, 2005; revised August 08, 2008. Current version published March 18, 2009. This work was supported in part by CTA-ARL DAAD 190120011.

W. Su is with the Department of Electrical Engineering, State University of New York (SUNY) at Buffalo, NY 14260 USA (e-mail: weifeng@eng.buffalo. edu).

Z. Safar is with the US R\&D Center, Samsung Electro-Mechanics America, Inc., Atlanta, GA 30332 USA (e-mail: zoltan.safar@ samsung.com).

K. J. R. Liu is with the Department of Electrical and Computer Engineering, University of Maryland, College Park, MD 20742 USA (e-mail: kjrliu@eng. umd.edu)

Communicated by E. Viterbo, Associate Editor for Coding Techniques.

Digital Object Identifier 10.1109/TIT.2009.2013027 transmit diversity scheme using orthogonal designs was proposed in [3] to construct STBCs for two transmit antennas from a $2 \times 2$ complex orthogonal design. The idea was extended in [4] to nonsquare code matrices and more transmit antennas. In [5], it was shown that for linear receivers, the orthogonal signaling structure is optimal in the sense that it maximizes the receiver signal to noise ratio. Results from the theory of amicable orthogonal designs were used to construct STBCs. The design of full-diversity, square, complex STBCs was considered in [6] with the aim to reduce the decoding delay and to maintain the maximum achievable symbol rate. The design procedure was based on the properties of the underlying Clifford algebra. There are different realizations of orthogonal designs [24], [5], [6]. A simple recursive expression for orthogonal designs was given in [7]. If nonsquare codewords are allowed, the transmission rates of orthogonal STBCs can be improved and a systematic code design method was presented in [8].

To increase the transmission rates, the authors of [9] and [10] also constructed STBCs from block-orthogonal designs which are also well known as quasi-orthogonal designs. In case of the block-orthogonal designs, the columns of the code matrices are grouped, and the columns within a group are not orthogonal, but the columns belonging to different groups are orthogonal to each other. This design approach increases the symbol rate, but the resulting STBCs, in general, cannot achieve full diversity. Fulldiversity block-orthogonal STBCs were proposed in [11]-[14]. The full-diversity property was ensured by taking some of the channel symbols from a rotated version of the used constellation with a carefully chosen rotation angle.

This work considers the problem of further improving the performance of STBCs. We propose a class of space-time codes constructed by combining orthogonal designs with sphere packing in a systematic way for an arbitrary number of transmit antennas. In case of the conventional STBCs from orthogonal designs, the symbols are chosen independently from a given constellation. The basic idea of our method is to determine the values of the symbols in the orthogonal designs jointly. The structure of the orthogonal designs is exploited to achieve full diversity, and sphere packing is used to improve the coding advantage. We also construct full-diversity STBCs from block-orthogonal designs with sphere packing. The full diversity is guaranteed by choosing some of the symbols from a rotated version of the used sphere packing. The optimal rotation angle that maximizes the normalized coding advantage will be determined for a class of sphere packing.

The paper is organized as follows. Section II will introduce the channel model and briefly summarize the relevant results from previous work. Section III will describe the STBC design method for orthogonal designs with sphere packing. The 
code construction method for block-orthogonal designs will be given in Section IV. The simulation results will be provided in Section V, and some conclusions will be drawn in the last section.

\section{Channel Model AND STBC Design CRITERIA}

We consider a wireless communication system with $M$ transmit antennas and $N$ receive antennas. The encoder divides the input bit stream into $b$ bit long blocks, and for each block, it selects one space-time codeword from the codeword set of size $L=2^{b}$. The selected codeword is then transmitted through the channel over the $M$ transmit antennas and $T$ time slots. Each codeword can be represented as a $T \times M$ matrix

$$
C=\left[\begin{array}{cccc}
c_{1}^{1} & c_{1}^{2} & \cdots & c_{1}^{M} \\
c_{2}^{1} & c_{2}^{2} & \cdots & c_{2}^{M} \\
\vdots & \vdots & \ddots & \vdots \\
c_{T}^{1} & c_{T}^{2} & \cdots & c_{T}^{M}
\end{array}\right]_{T \times M}
$$

where $c_{t}^{i}$ denotes the channel symbol transmitted by transmit antenna $i, i=1,2, \ldots, M$, at discrete time $t, t=1,2, \ldots, T$. The codewords are assumed to satisfy the energy constraint $E\|C\|_{F}^{2}=M T$, where $\|C\|_{F}$ is the Frobenius norm ${ }^{1}$ of $C$, and $E$ stands for the expectation.

The transmission medium is assumed to be flat (frequency nonselective), quasi-static, Rayleigh-fading channel, so the channel stays constant during the transmission of one codeword. The MIMO transceiver can be modeled as

$$
Y=\sqrt{\frac{\rho}{M}} C H+Z
$$

where $Y=\left\{y_{t}^{j}\right\}_{1 \leq t \leq T, 1 \leq j \leq N}$ is the received signal matrix of size $T \times N$ in which $y_{t}^{j}$ is the received signal at receive antenna $j$ at time $t, H=\left\{h_{i, j}\right\}_{1 \leq i \leq M, 1 \leq j \leq N}$ is the channel coefficient matrix of size $M \times N$ in which $h_{i, j}$ is the channel coefficient between transmit antenna $i$ and receive antenna $j, Z=$ $\left\{z_{t}^{j}\right\}_{1 \leq t \leq T, 1 \leq j \leq N}$ is the noise matrix of size $T \times N$, and $C$ is the space-time codeword, as defined in (1). The channel coefficients and noise are modeled as zero-mean, complex Gaussian random variables with unit variance. The factor $\sqrt{\rho / M}$ in (2) ensures that $\rho$ is the average SNR at each receive antenna, and it is independent of the number of transmit antennas.

Assuming that the channel matrix $H$ is available at the receiver, the maximum likelihood decoding algorithm chooses the decoded codeword $\tilde{C}$ according to $\tilde{C}=\arg \min _{C} \| Y-$ $\sqrt{\frac{\rho}{M}} C H \|_{F}^{2}$. The pairwise error probability between two distinct codewords $C$ and $\tilde{C}$ can be upper bounded as [1], [2]

$$
P(C \rightarrow \tilde{C}) \leq \frac{1}{2}\left(\prod_{i=1}^{r} \lambda_{i}\right)^{-N} \cdot\left(\frac{\rho}{4 M}\right)^{-r N}
$$

where $r=\operatorname{rank}(C-\tilde{C})$, and $\lambda_{1}, \lambda_{2}, \ldots, \lambda_{r}$ are the nonzero eigenvalues of $(C-\tilde{C})(C-\tilde{C})^{\mathcal{H}}$. The superscript $\mathcal{H}$ stands for

${ }^{1}$ The Frobenius norm of $C$ is defined as

$$
\|C\|_{F}^{2}=\operatorname{tr}\left(C^{\mathcal{H}} C\right)=\operatorname{tr}\left(C C^{\mathcal{H}}\right)=\sum_{t=1}^{T} \sum_{i=1}^{M}\left|c_{t}^{i}\right|^{2} .
$$

the complex conjugate and transpose of a matrix. Based on the pairwise error probability, two code design criteria have been proposed [1], [2]: 1) The minimum rank of the code difference matrix $C-\tilde{C}$ over all distinct codewords $C$ and $\tilde{C}$ should be as large as possible; and 2) The minimum value of the product $\prod_{i=1}^{r} \lambda_{i}$ over all distinct codewords $C$ and $\tilde{C}$ should be as large as possible. This quantity is referred to as the coding advantage achieved by the STBC. If the difference matrix $C-\tilde{C}$ is always of full rank, the objective is to maximize the determinant of $(C-\tilde{C})^{\mathcal{H}}(C-\tilde{C})$.

If the matrix $C-\tilde{C}$ is always of full rank for a specific STBC, we say that this STBC achieves full diversity. We consider the design of STBCs of square size, i.e., $T=M$. If a STBC of square size achieves full diversity, the diversity product, which is the normalized coding advantage, is given by [15], [16]

$$
\zeta=\frac{1}{2 \sqrt{M}} \min _{C \neq \tilde{C}}|\operatorname{det}(C-\tilde{C})|^{1 / M} .
$$

Substituting (4) into (3), we obtain $P(C \rightarrow \tilde{C}) \leq \frac{1}{2}\left(\zeta^{2} \rho\right)^{-M N}$, so it is desirable to maximize the diversity product $\zeta$ if the full diversity has been achieved. In [17], it was shown that for a STBC consisting of $L$ codewords, the diversity product is upper bounded by $\zeta \leq \sqrt{\frac{L}{2(L-1)}}$.

\section{ORThogonal Designs With Sphere PACKING}

In this section, we consider the construction of STBCs from orthogonal designs with sphere packing for $M=2^{k}$ $(k=1,2,3, \ldots)$ transmit antennas. If the number of transmit antennas is not a power of two, the desired STBC can be obtained by deleting some columns from a larger STBC designed for a power of two antennas. The structure of the orthogonal designs will be exploited to achieve full diversity, and sphere packing will be used to maximize the diversity product (coding advantage).

A recursive expression for orthogonal designs was given in [7] as follows. Let $G_{1}\left(x_{1}\right)=x_{1} I_{1}$, and

$$
\begin{aligned}
G_{2^{k}} & \left(x_{1}, \ldots, x_{k+1}\right) \\
= & {\left[\begin{array}{cc}
G_{2^{k-1}}\left(x_{1}, \ldots, x_{k}\right) & x_{k+1} I_{2^{k-1}} \\
-x_{k+1}^{*} I_{2^{k-1}} & G_{2^{k-1}}^{\mathcal{H}}\left(x_{1}, \ldots, x_{k}\right)
\end{array}\right] } \\
& k=1,2,3, \ldots .
\end{aligned}
$$

Then, $G_{2^{k}}\left(x_{1}, x_{2}, \ldots, x_{k+1}\right)$ is an orthogonal design of size $2^{k} \times 2^{k}$ with complex variables $x_{1}, x_{2}, \ldots, x_{k+1}$. The symbol rate of $G_{2^{k}}$ is $(k+1) / 2^{k}$, which is the maximum rate for orthogonal designs of square size ([5]-[7] and the references therein).

For $M=2^{k}$ transmit antennas, STBCs can be constructed from the orthogonal design $G_{2^{k}}$ as

$$
C=\sqrt{2^{k} /(k+1)} G_{2^{k}}\left(x_{1}, x_{2}, \ldots, x_{k+1}\right)
$$

with some specific choices of $x_{1}, x_{2}, \ldots, x_{k+1}$ such that $E\left(\left|x_{1}\right|^{2}+\left|x_{2}\right|^{2}+\cdots+\left|x_{k+1}\right|^{2}\right)=k+1$. For example, if the complex variables $x_{1}, x_{2}, \ldots, x_{k+1}$ are chosen independently from a QPSK constellation, there are totally $L=4^{k+1}$ codewords to be transmitted. The normalization factor $\sqrt{2^{k} /(k+1)}$ in (6) ensures that $C$ satisfies the energy 
constraint $E\|C\|_{F}^{2}=M T=2^{2 k}$. In case of the conventional STBCs from orthogonal designs, the symbols in the orthogonal designs are chosen independently from PSK or QAM constellations. The basic idea of the new scheme is that we design these symbols jointly with sphere packing to further increase the coding advantage.

For two distinct codewords

and

$$
C=\sqrt{\frac{2^{k}}{k+1}} G_{2^{k}}\left(x_{1}, x_{2}, \ldots, x_{k+1}\right)
$$

$$
\tilde{C}=\sqrt{\frac{2^{k}}{k+1}} G_{2^{k}}\left(\tilde{x}_{1}, \tilde{x}_{2}, \ldots, \tilde{x}_{k+1}\right)
$$

the code difference matrix is given by

$$
C-\tilde{C}=\sqrt{\frac{2^{k}}{k+1}} G_{2^{k}}\left(x_{1}-\tilde{x}_{1}, x_{2}-\tilde{x}_{2}, \ldots, x_{k+1}-\tilde{x}_{k+1}\right)
$$

and

$$
\begin{aligned}
(C-\tilde{C})(C-\tilde{C})^{\mathcal{H}} & =(C-\tilde{C})^{\mathcal{H}}(C-\tilde{C}) \\
& =\frac{2^{k}}{k+1}\left(\sum_{i=1}^{k+1}\left|x_{i}-\tilde{x}_{i}\right|^{2}\right) I_{2^{k}} .(7)
\end{aligned}
$$

It is easy to see that for two different vectors $\left(x_{1}, x_{2}, \ldots, x_{k+1}\right)$ and $\left(\tilde{x}_{1}, \tilde{x}_{2}, \ldots, \tilde{x}_{k+1}\right)$, the full rank of the code difference matrix is guaranteed.

Having ensured that the STBCs achieve full diversity, the next step is to maximize the diversity product. From (4), the diversity product can be expressed as

$\zeta=\frac{1}{2 \sqrt{k+1}} \min _{\left(x_{1}, \ldots, x_{k+1}\right) \neq\left(\tilde{x}_{1}, \ldots, \tilde{x}_{k+1}\right)}\left(\sum_{i=1}^{k+1}\left|x_{i}-\tilde{x}_{i}\right|^{2}\right)^{\frac{1}{2}}$.

As a consequence, the diversity product is determined by the minimum Euclidean distance of the set of $(k+1)$-dimensional complex vectors $\left\{\left(x_{1}, x_{2}, \ldots, x_{k+1}\right)\right\}$ underlying the STBC. Therefore, sphere packing in the $2(k+1)$-dimensional real Euclidean space $\mathrm{R}^{2(k+1)}$ [29] can be used to maximize the diversity product. More formally, assume that $S=\left\{\left[a_{l, 1} a_{l, 2} \ldots a_{l, 2(k+1)}\right]^{T} \in \mathrm{R}^{2(k+1)}: 0 \leq l \leq L-1\right\}$ is a set of $L$ points from a $2(k+1)$-dimensional sphere packing with total energy $\sum_{l=0}^{L-1}\left(\left|a_{l, 1}\right|^{2}+\left|a_{l, 2}\right|^{2}+\cdots+\left|a_{l, 2(k+1)}\right|^{2}\right) \triangleq E$. For each vector in $S$, we can define a corresponding set of complex symbols $\left\{x_{i}\right\}_{1 \leq i \leq k+1}$ as: $x_{i}=a_{l, 2 i-1}+\mathbf{j} a_{l, 2 i}$, where $\mathbf{j} \triangleq \sqrt{-1}$. Then, the matrices

$$
C_{l}=\sqrt{2^{k} L / E} G_{2^{k}}\left(x_{1}, x_{2}, \ldots, x_{k+1}\right), \quad l=0,1, \ldots, L-1
$$

form a set of space-time codewords whose diversity product is determined by the minimum Euclidean distance of $S$. The factor $\sqrt{2^{k} L / E}$ ensures that the resulting STBC satisfies the energy constraint (assuming that all codewords are equally likely to be transmitted).

In the following sections, we will provide code design examples for two and four transmit antennas.

\section{A. Code Design for Two Transmit Antennas}

For two transmit antennas, the $2 \times 2$ orthogonal design is

$$
G_{2}\left(x_{1}, x_{2}\right)=\left[\begin{array}{cc}
x_{1} & x_{2} \\
-x_{2}^{*} & x_{1}^{*}
\end{array}\right]
$$

which was first used by Alamouti in space-time coding [3]. Later in [16], a similar structure with constraint $\left|x_{1}\right|^{2}+\left|x_{2}\right|^{2}=1$ was used to build $2 \times 2$ unitary matrices, the so-called Hamiltonian constellation for differential modulation. This constraint is not necessary for the design of STBCs with coherent detection.

In the four-dimensional real Euclidean space $\mathrm{R}^{4}$, the sphere packing with the best known minimum Euclidean distance is a lattice that consists of all points with integer coordinates $\left(a_{1}, a_{2}, a_{3}, a_{4}\right)$ such that $a_{1}+a_{2}+a_{3}+a_{4}$ is even [29], and it is usually denoted as $D_{4}$. Alternatively, $D_{4}$ may also be defined as the integer span of the vectors $v_{1}, v_{2}, v_{3}, v_{4}$ that form the rows of the generator matrix

$$
\left[\begin{array}{llll}
2 & 0 & 0 & 0 \\
1 & 1 & 0 & 0 \\
1 & 0 & 1 & 0 \\
1 & 0 & 0 & 1
\end{array}\right]
$$

We now combine the orthogonal design $G_{2}$ and the sphere packing $D_{4}$ to construct STBCs for two transmit antennas. Assume that $S=\left\{\left[a_{l, 1} a_{l, 2} a_{l, 3} a_{l, 4}\right]^{T} \in \mathrm{R}^{4}: 0 \leq l \leq L-1\right\}$ is a set of $L$ points from $D_{4}$ with total energy $\sum_{l=0}^{L-1}\left(\left|a_{l, 1}\right|^{2}+\right.$ $\left.\left|a_{l, 2}\right|^{2}+\left|a_{l, 3}\right|^{2}+\left|a_{l, 4}\right|^{2}\right) \triangleq E$. Let

$$
\begin{aligned}
C_{l} & =\sqrt{2 L / E} G_{2}\left(a_{l, 1}+\mathbf{j} a_{l, 2}, a_{l, 3}+\mathbf{j} a_{l, 4}\right) \\
l & =0,1, \ldots, L-1 .
\end{aligned}
$$

Then, $\left\{C_{l}: 0 \leq l \leq L-1\right\}$ is a set of space-time codewords whose diversity product is determined by the minimum Euclidean distance of $S$.

We list the diversity products of the orthogonal designs with sphere packing (abbreviated as Orth. with S.P.) for two transmit antennas in Table I, and compare them with those of the cyclic codes [15], the parametric codes [17], the diagonal algebraic codes [21], the high rate codes $B_{2}(\phi, \theta)$ [20], and the orthogonal designs with PSK or QAM constellations. Table I shows that the diversity product of the orthogonal designs with sphere packing is greater than that of the other schemes except the case of $L=$ 16 and $L=256$. The diversity product of the diagonal algebraic codes is the same as that of orthogonal designs with the same QAM constellations. Note that for $L=4$, there are optimal space-time block codes [17], [18], in the sense that the diversity product achieves the upper bound $\sqrt{2 / 3}$, and they can be shown to be equivalent to the codes obtained by the proposed method.

\section{B. Code Design for Four Transmit Antennas}

The $4 \times 4$ orthogonal design for four transmit antennas is given by

$$
G_{4}\left(x_{1}, x_{2}, x_{3}\right)=\left[\begin{array}{cccc}
x_{1} & x_{2} & x_{3} & 0 \\
-x_{2}^{*} & x_{1}^{*} & 0 & x_{3} \\
-x_{3}^{*} & 0 & x_{1}^{*} & -x_{2} \\
0 & -x_{3}^{*} & x_{2}^{*} & x_{1}
\end{array}\right] .
$$


TABLE I

Comparison of Diversity Product for Two Transmit Antennas. Note That We Abbreviate Orthogonal Designs WITH SPHERE PACKING AS ORTH. WITH S.P.

\begin{tabular}{|c|c|c|c|c|}
\hline $\begin{array}{c}\text { Size } \\
L\end{array}$ & $\begin{array}{c}\text { Rate } \\
\text { (bits/s/Hz) }\end{array}$ & $\begin{array}{c}\text { Upper bound } \\
\sqrt{\frac{L}{2(L-1)}}\end{array}$ & $\begin{array}{c}\text { Diversity product } \\
\zeta \\
\end{array}$ & Comments \\
\hline 4 & 1 & 0.8165 & $\begin{array}{l}0.7071 \\
0.7071 \\
0.7071 \\
0.8165\end{array}$ & $\begin{array}{l}\text { Cyclic code } \\
\text { Diag. algebr. code } \\
\text { Orth. with BPSK } \\
\text { Orth. with S.P. }\end{array}$ \\
\hline 8 & 1.5 & 0.7559 & $\begin{array}{l}0.5946 \\
0.7071 \\
0.7071\end{array}$ & $\begin{array}{l}\text { Cyclic code } \\
\text { Parametric code } \\
\text { Orth. with S.P. }\end{array}$ \\
\hline 16 & 2 & 0.7303 & $\begin{array}{l}0.3827 \\
0.5946 \\
0.5000 \\
0.5000 \\
0.4729 \\
0.5535\end{array}$ & $\begin{array}{l}\text { Cyclic code } \\
\text { Parametric code } \\
\text { Diag. algebr. code } \\
\text { Orth. with QPSK } \\
\text { Golden code with BPSK } \\
\text { Orth. with S.P. }\end{array}$ \\
\hline 32 & 2.5 & 0.7184 & $\begin{array}{l}0.2494 \\
0.3827 \\
0.4658\end{array}$ & $\begin{array}{l}\text { Cyclic code } \\
\text { Parametric code } \\
\text { Orth. with S.P. }\end{array}$ \\
\hline 64 & 3 & 0.7127 & $\begin{array}{l}0.1985 \\
0.3070 \\
0.2706 \\
0.3162 \\
0.3860\end{array}$ & $\begin{array}{l}\text { Cyclic code } \\
\text { Parametric code } \\
\text { Orth. with 8PSK } \\
\text { Orth. with 8QAM } \\
\text { Orth. with S.P. }\end{array}$ \\
\hline 128 & 3.5 & 0.7099 & $\begin{array}{l}0.1498 \\
0.2606 \\
0.3226\end{array}$ & $\begin{array}{l}\text { Cyclic code } \\
\text { Parametric code } \\
\text { Orth. with S.P. }\end{array}$ \\
\hline 256 & 4 & 0.7085 & $\begin{array}{l}0.0988 \\
0.2237 \\
0.2544 \\
0.3344 \\
0.2237 \\
0.2722\end{array}$ & $\begin{array}{l}\text { Cyclic code } \\
\text { Diag. algebr. code } \\
B_{2}(\phi, \theta) \text { with QPSK } \\
\text { Golden code with 4QAM } \\
\text { Orth. with 16QAM } \\
\text { Orth. with S.P. }\end{array}$ \\
\hline 512 & 4.5 & 0.7078 & $\begin{array}{l}0.0697 \\
0.2285\end{array}$ & $\begin{array}{l}\text { Cyclic code } \\
\text { Orth. with S.P. }\end{array}$ \\
\hline 1024 & 5 & 0.7075 & $\begin{array}{l}0.0481 \\
0.1581 \\
0.1581 \\
0.1918\end{array}$ & $\begin{array}{l}\text { Cyclic code } \\
\text { Diag. algebr. code } \\
\text { Orth. with 32QAM } \\
\text { Orth. with S.P. }\end{array}$ \\
\hline
\end{tabular}

The symbol rate of $G_{4}$ is $3 / 4$. We consider sphere packing in six-dimensional real Euclidean space $\mathrm{R}^{6}$ since there are three complex symbols $x_{1}, x_{2}, x_{3}$ in $G_{4}$.

In $\mathrm{R}^{6}$, the sphere packing with the best known minimum Euclidean distance is the lattice $E_{6}$ [29]. $E_{6}$ has a simple description as a three-dimensional complex lattice over the Eisenstein integers. ${ }^{2}$ Precisely, $E_{6}$ is the Eisenstein integer span of the vectors $e_{1}, e_{2}, e_{3}$ that form the rows of the generator matrix $\left[\begin{array}{lll}\theta & 0 & 0 \\ 0 & \theta & 0 \\ 1 & 1 & 1\end{array}\right]$, where $\theta=\mathbf{j} \sqrt{3}$.

We now combine the sphere packing $E_{6}$ and the orthogonal design $G_{4}$ to construct STBCs for four transmit antennas. Assume that $S=\left\{\left[a_{l, 1} a_{l, 2} \ldots a_{l, 6}\right]^{T} \in \mathrm{R}^{6}: 0 \leq l \leq L-1\right\}$ is a set of $L$ points from $E_{6}$ with total energy

$$
\sum_{l=0}^{L-1}\left(\left|a_{l, 1}\right|^{2}+\left|a_{l, 2}\right|^{2}+\cdots+\left|a_{l, 6}\right|^{2}\right) \triangleq E .
$$

${ }^{2}$ The set of Eisenstein integers is defined as [29, p. 52],

$$
\mathcal{E}=\{k+\omega l: \text { both } k \text { and } l \text { are integers }\}
$$

in which $\omega=(-1+\mathbf{j} \sqrt{3}) / 2$. Note that the hexagonal lattice modulation is corresponding to a one-dimensional lattice over the Eisenstein integers.
Let

$$
\begin{array}{r}
C_{l}=\sqrt{4 L / E} G_{4}\left(a_{l, 1}+\mathbf{j} a_{l, 2}, a_{l, 3}+\mathbf{j} a_{l, 4}, a_{l, 5}+\mathbf{j} a_{l, 6}\right) \\
l=0,1, \ldots, L-1 .
\end{array}
$$

Then, $\left\{C_{l}: 0 \leq l \leq L-1\right\}$ is a set of space-time codewords whose diversity product is determined by the minimum Euclidean distance of $S$. We list the diversity products of the orthogonal designs with sphere packing for four transmit antennas in Table II, and compare them with those of the cyclic codes [15], the orthogonal designs with PSK constellations, the diagonal algebraic codes [21], and the full-diversity block-orthogonal designs [13], [14]. We can see that the diversity products of the orthogonal designs with sphere packing are greater than those of other four schemes. Note that the diversity product of the diagonal algebraic codes is the same as that of the full-diversity block-orthogonal design in case of using the same QAM constellations. For $L=4$, the diversity product of the proposed scheme achieves the upper bound 0.8165 .

\section{Block-Orthogonal Designs With Sphere Packing}

In case of four or more transmit antennas, there are STBCs from block-orthogonal designs [9], [10] that can provide higher 
TABLE II

Comparison of Diversity Product for Four Transmit Antennas. Note That We Abbreviate Orthogonal Designs With SPHERE Packing as Orth. With S.P., AND Block-Orthogonal Designs With Sphere Packing As Block-Orth. With S.P.

\begin{tabular}{|c|c|c|c|c|}
\hline $\begin{array}{c}\text { Size } \\
L\end{array}$ & $\begin{array}{c}\text { Rate } \\
\text { (bits/s/Hz) }\end{array}$ & $\begin{array}{c}\text { Upper bound } \\
\sqrt{\frac{L}{2(L-1)}}\end{array}$ & $\begin{array}{c}\text { Diversity product } \\
\zeta\end{array}$ & Comments \\
\hline 4 & 0.5 & 0.8165 & $\begin{array}{l}0.7071 \\
0.8165\end{array}$ & $\begin{array}{l}\text { Cyclic code } \\
\text { Orth. with S.P. }\end{array}$ \\
\hline 8 & 0.75 & 0.7559 & $\begin{array}{l}0.5946 \\
0.5774 \\
0.7184\end{array}$ & $\begin{array}{l}\text { Cyclic code } \\
\text { Orth. with BPSK } \\
\text { Orth. with S.P. }\end{array}$ \\
\hline 16 & 1 & 0.7303 & $\begin{array}{l}0.5453 \\
0.5000 \\
0.6426 \\
0.5000 \\
0.5774\end{array}$ & $\begin{array}{l}\text { Cyclic code } \\
\text { Diag. algebr. code } \\
\text { Orth. with S.P. } \\
\text { Block-Orth. with BPSK } \\
\text { Block-Orth. with S.P. }\end{array}$ \\
\hline 32 & 1.25 & 0.7184 & $\begin{array}{l}0.3827 \\
0.5611\end{array}$ & $\begin{array}{l}\text { Cyclic code } \\
\text { Orth. with S.P. }\end{array}$ \\
\hline 64 & 1.5 & 0.7127 & $\begin{array}{l}0.3399 \\
0.4083 \\
0.5062\end{array}$ & $\begin{array}{l}\text { Cyclic code } \\
\text { Orth. with QPSK } \\
\text { Orth. with S.P. }\end{array}$ \\
\hline 128 & 1.75 & 0.7099 & $\begin{array}{l}0.2594 \\
0.4390\end{array}$ & $\begin{array}{l}\text { Cyclic code } \\
\text { Orth. with S.P. }\end{array}$ \\
\hline 256 & 2 & 0.7085 & $\begin{array}{l}0.2208 \\
0.3536 \\
0.3884 \\
0.3536 \\
0.3885\end{array}$ & $\begin{array}{l}\text { Cyclic code } \\
\text { Diag. algebr. code } \\
\text { Orth. with S.P. } \\
\text { Block-Orth. with QPSK } \\
\text { Block-Orth. with S.P. }\end{array}$ \\
\hline 512 & 2.25 & 0.7078 & $\begin{array}{l}0.1940 \\
0.2210 \\
0.3467\end{array}$ & $\begin{array}{l}\text { Cyclic code } \\
\text { Orth. with 8PSK } \\
\text { Orth. with S.P. }\end{array}$ \\
\hline 1024 & 2.5 & 0.7075 & $\begin{array}{l}0.1532 \\
0.3105\end{array}$ & $\begin{array}{l}\text { Cyclic code } \\
\text { Orth. with S.P. }\end{array}$ \\
\hline 4096 & 3 & 0.7072 & $\begin{array}{l}0.2390 \\
0.1826 \\
0.2454 \\
0.2236 \\
0.2729\end{array}$ & $\begin{array}{l}\text { Diag. algebr. code } \\
\text { Orth. with 16QAM } \\
\text { Orth. with S.P. } \\
\text { Block-Orth. with 8QAM } \\
\text { Block-Orth. with S.P. }\end{array}$ \\
\hline
\end{tabular}

symbol transmission rate than those from orthogonal designs. In order to obtain higher coding advantage, we also consider STBC construction from block-orthogonal designs with sphere packing. First, we will provide the code design method for four transmit antennas, and then we will describe the general case for any power of two transmit antennas.

\section{A. Code Design for Four Transmit Antennas}

For four transmit antennas, a STBC with symbol transmission rate 1 was constructed [10] from the $2 \times 2$ Alamouti scheme as follows:

$$
\begin{aligned}
Q_{4}\left(x_{1}, x_{2}, x_{3}, x_{4}\right) & =\left[\begin{array}{cc}
A & B \\
B & A
\end{array}\right] \\
& =\left[\begin{array}{cccc}
x_{1} & x_{2} & x_{3} & x_{4} \\
-x_{2}^{*} & x_{1}^{*} & -x_{4}^{*} & x_{3}^{*} \\
x_{3} & x_{4} & x_{1} & x_{2} \\
-x_{4}^{*} & x_{3}^{*} & -x_{2}^{*} & x_{1}^{*}
\end{array}\right]
\end{aligned}
$$

where $A=\left[\begin{array}{cc}x_{1} & x_{2} \\ -x_{2}^{*} & x_{1}^{*}\end{array}\right], B=\left[\begin{array}{cc}x_{3} & x_{4} \\ -x_{4}^{*} & x_{3}^{*}\end{array}\right]$, and $x_{1}, x_{2}, x_{3}, x_{4}$ are chosen from some signal constellations. In general, this scheme is not guaranteed to achieve full diversity. However, by properly choosing the signal constellations, the full diversity can be achieved [13], [14]: $x_{1}$ and $x_{2}$ can be chosen from any constellation $\mathcal{A}$, and $x_{3}$ and $x_{4}$ can be chosen from the rotated constellation $e^{\mathbf{j} \phi} \mathcal{A}$.

We now construct block-orthogonal STBCs with sphere packing for four transmit antennas from the block-orthogonal design of (14), but the proposed method can be extended easily to other block-orthogonal structures. We combine the block-orthogonal design $Q_{4}$ and the sphere packing $D_{4}$ in the following way. Assume that $S=\left\{\left[a_{l, 1} a_{l, 2} a_{l, 3} a_{l, 4}\right] \in \mathrm{R}^{4}\right.$ : $\left.0 \leq l \leq L_{0}-1\right\}$ is a set of $L_{0}$ points from $D_{4}$ with total energy $\sum_{l=0}^{L_{0}-1}\left(\left|a_{l, 1}\right|^{2}+\left|a_{l, 2}\right|^{2}+\left|a_{l, 3}\right|^{2}+\left|a_{l, 4}\right|^{2}\right) \triangleq E$. Let

$$
\begin{aligned}
& x_{1}=a_{l, 1}+\mathbf{j} a_{l, 2} \\
& x_{2}=a_{l, 3}+\mathbf{j} a_{l, 4}, \quad 0 \leq l \leq L_{0}-1 \\
& x_{3}=e^{\mathbf{j} \phi}\left(a_{l^{\prime}, 1}+\mathbf{j} a_{l^{\prime}, 2}\right) \\
& x_{4}=e^{\mathbf{j} \phi}\left(a_{l^{\prime}, 3}+\mathbf{j} a_{l^{\prime}, 4}\right), \quad 0 \leq l^{\prime} \leq L_{0}-1 .
\end{aligned}
$$

If we define

$$
C_{l, l^{\prime}}=\sqrt{\frac{2 L_{0}}{E}} Q_{4}\left(x_{1}, x_{2}, x_{3}, x_{4}\right)
$$

then $\left\{C_{l, l^{\prime}}: 0 \leq l, l^{\prime} \leq L_{0}-1\right\}$ is a STBC with $L=L_{0}^{2}$ codewords. In fact, the symbols $x_{1}$ and $x_{2}$ are taken from the sphere packing $S$ in the same way as described in Section III-A, and the symbols $x_{3}$ and $x_{4}$ are taken from the sphere packing 


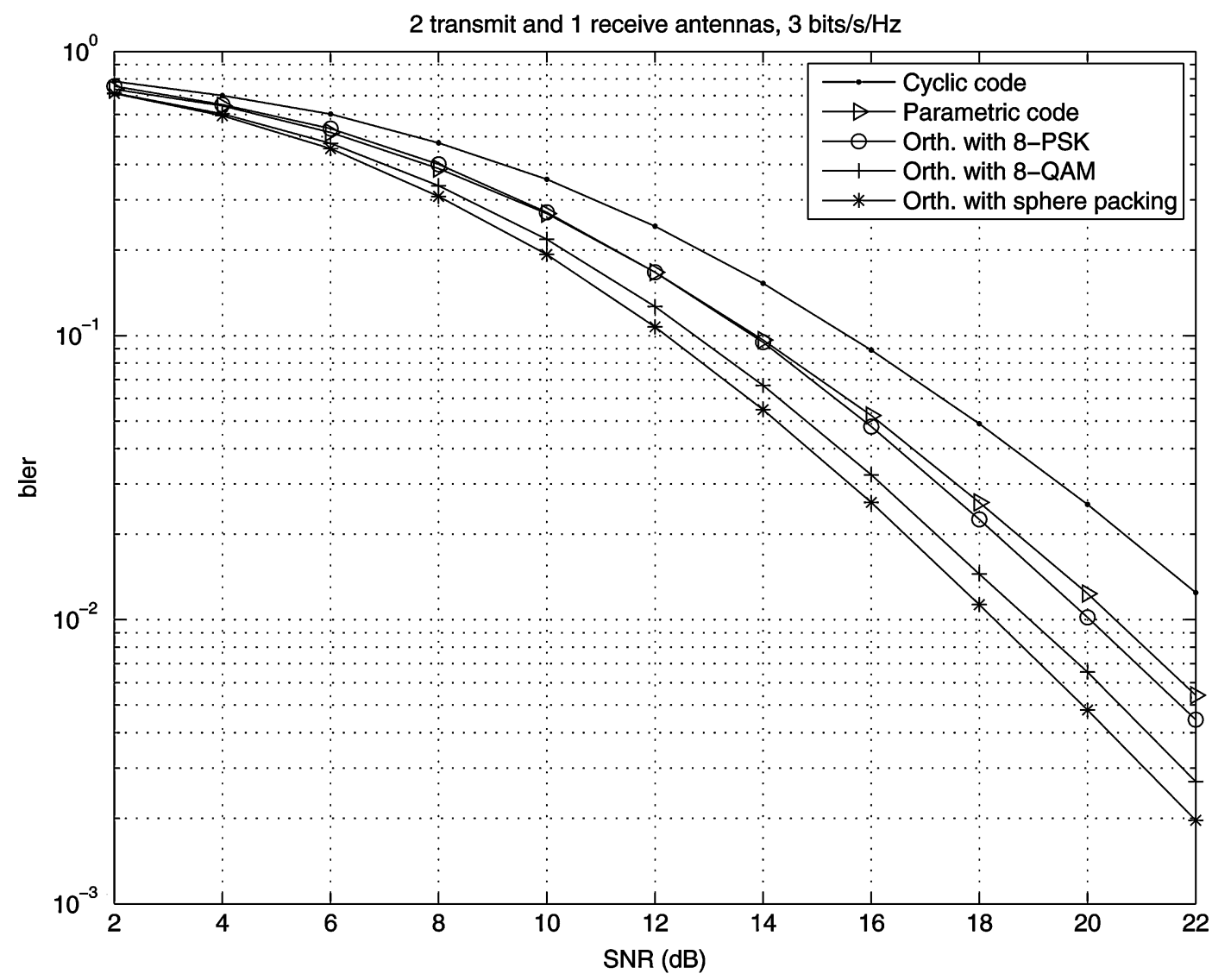

Fig. 1. Block error rate performance of cyclic code “.," parametric code " $\triangleright$, , orth. with 8 PSK “o," orth. with 8 QAM “+," and orth. with sphere packing “*." $L=64$.

$S^{\prime} \in \mathrm{R}^{4}$. The set of points $S^{\prime}$ is a rotated version of $S$ since for each $\left[a_{l, 1} a_{l, 2} a_{l, 3} a_{l, 4}\right]^{T} \in S$, there is a corresponding $\left[a_{l, 1}^{\prime} a_{l, 2}^{\prime} a_{l, 3}^{\prime} a_{l, 4}^{\prime}\right]^{T} \in S^{\prime}$ such that

$$
\left[\begin{array}{l}
a_{l, 1}^{\prime} \\
a_{l, 2}^{\prime} \\
a_{l, 3}^{\prime} \\
a_{l, 4}^{\prime}
\end{array}\right]=\left[\begin{array}{cccc}
\cos \phi & -\sin \phi & 0 & 0 \\
\sin \phi & \cos \phi & 0 & 0 \\
0 & 0 & \cos \phi & -\sin \phi \\
0 & 0 & \sin \phi & \cos \phi
\end{array}\right]\left[\begin{array}{l}
a_{l, 1} \\
a_{l, 2} \\
a_{l, 3} \\
a_{l, 4}
\end{array}\right] .
$$

The factor $\sqrt{2 L_{0} / E}$ in (15) ensures that the resulting STBC satisfies the energy constraint. The diversity product can be calculated as [14]

$$
\begin{aligned}
\zeta= & \frac{1}{2} \sqrt{\frac{L_{0}}{2 E}} \min _{X \neq \tilde{X}} \\
& \times\left(\sum_{i=1}^{2}\left|\left(x_{i}-\tilde{x}_{i}\right)+\left(x_{2+i}-\tilde{x}_{2+i}\right)\right|^{2}\right)^{1 / 4} \\
& \times\left(\sum_{i=1}^{2}\left|\left(x_{i}-\tilde{x}_{i}\right)-\left(x_{2+i}-\tilde{x}_{2+i}\right)\right|^{2}\right)^{1 / 4}
\end{aligned}
$$

where $X=\left(x_{1}, x_{2}, x_{3}, x_{4}\right)$ and $\tilde{X}=\left(\tilde{x}_{1}, \tilde{x}_{2}, \tilde{x}_{3}, \tilde{x}_{4}\right)$. Let

and

$$
\left[\begin{array}{l}
u_{1} \\
u_{3}
\end{array}\right]=\frac{1}{\sqrt{2}}\left[\begin{array}{cc}
1 & 1 \\
1 & -1
\end{array}\right]\left[\begin{array}{l}
x_{1} \\
x_{3}
\end{array}\right]
$$

$$
\left[\begin{array}{l}
u_{2} \\
u_{4}
\end{array}\right]=\frac{1}{\sqrt{2}}\left[\begin{array}{cc}
1 & 1 \\
1 & -1
\end{array}\right]\left[\begin{array}{l}
x_{2} \\
x_{4}
\end{array}\right]
$$

then the diversity product in (16) becomes

$$
\begin{aligned}
\zeta=\frac{1}{2} \sqrt{\frac{L_{0}}{E}} \min _{U \neq \tilde{U}}\left(\mid u_{1}\right. & \left.-\left.\tilde{u}_{1}\right|^{2}+\left|u_{2}-\tilde{u}_{2}\right|^{2}\right)^{1 / 4} \\
& \times\left(\left|u_{3}-\tilde{u}_{3}\right|^{2}+\left|u_{4}-\tilde{u}_{4}\right|^{2}\right)^{1 / 4}
\end{aligned}
$$

where $U=\left(u_{1}, u_{2}, u_{3}, u_{4}\right)$ and $\tilde{U}=\left(\tilde{u}_{1}, \tilde{u}_{2}, \tilde{u}_{3}, \tilde{u}_{4}\right)$. This form allows us to use the results of [19] directly to determine the value of the rotation angle $\phi$. Since we choose $\left(x_{1}, x_{2}\right)$ from the sphere packing $S$ and choose $\left(x_{3}, x_{4}\right)$ from a rotated version of $S$, it was shown in [19] that if the rotation angle is chosen as $\phi=\pi / 4$, the diversity product in (16) or (17) is not zero, so the obtained STBC is guaranteed to achieve full diversity. Moreover, in case of the sphere packing $D_{4}$, the rotation angle $\phi=\pi / 4$ is optimal in the sense that we cannot choose any other rotation angle to obtain a higher diversity product ([19, p. 947]). We list the diversity products of the block-orthogonal designs with sphere packing $D_{4}$ for four transmit antennas in Table II. We can see that the diversity product can be increased by using block-orthogonal designs with sphere packing for large $L$, for example, $L=4096$. However, there is little or no advantage by using block-orthogonal designs with sphere packing in case of $L=16$ and 256 .

\section{B. Code Design for $M=2^{k+1}(k \geq 1)$ Transmit Antennas}

In this subsection, we consider the code design problem for $M=2^{k+1}(k \geq 1)$ transmit antennas. In case of $M=4(k=1)$, 


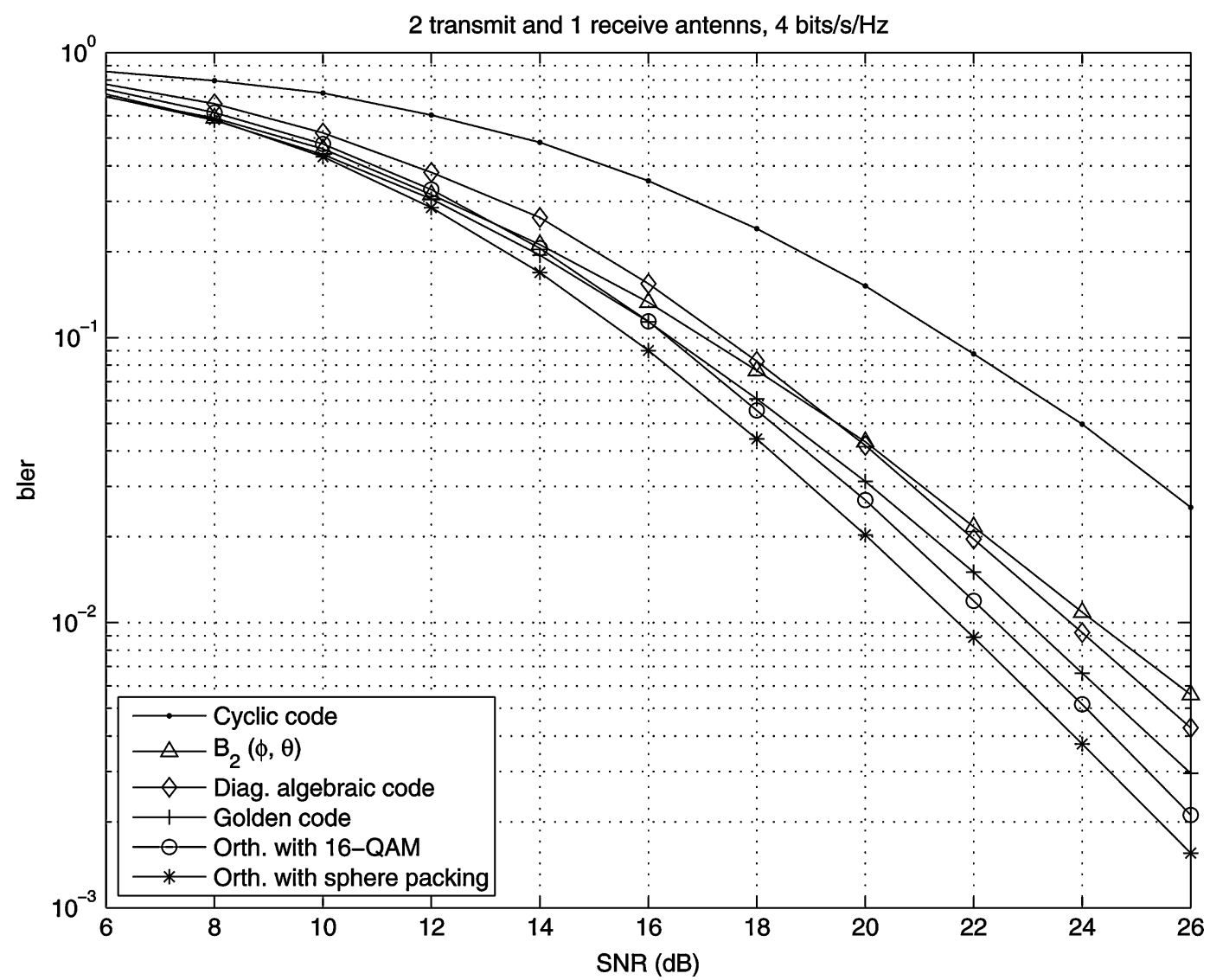

Fig. 2. Block error rate performance of cyclic code ".," diagonal algebraic code “ $\diamond, " B_{2}(\phi, \theta)[20]$ " $\triangle$," the Golden code “+," orth. with 16-QAM "o," and orth. with sphere packing " $* . " ~ L=256$.

the optimal rotation angle was obtained by taking advantage of the result in [19]. However, [19] does not offer a solution for the general case, so we have to develop a new method to determine the optimal value of $\phi$.

In general, for any orthogonal design $G_{2^{k}}\left(x_{1}, x_{2}, \ldots, x_{k+1}\right)$ given by (5), a block-orthogonal design $Q_{2^{k+1}}\left(x_{1}, x_{2}, \ldots\right.$, $\left.x_{2(k+1)}\right)$ of size $2^{k+1}$ by $2^{k+1}$ can be constructed as shown in (18) at the bottom of the page [9], [10], [13]. For any sphere packing

$$
S=\left\{\left[a_{l, 1} a_{l, 2} \cdots a_{l, 2(k+1)}\right]^{T} \in \mathrm{R}^{2(k+1)}: 0 \leq l \leq L_{0}-1\right\}
$$

with total energy $\sum_{l=0}^{L_{0}-1} \sum_{i=1}^{2(k+1)}\left|a_{l, i}\right|^{2} \triangleq E$, we combine the block-orthogonal design $Q_{2^{k+1}}$ and the sphere packing $S$ to construct STBCs for $M=2^{k+1}$ transmit antennas. For each $l\left(0 \leq l \leq L_{0}-1\right)$, let

$$
x_{i}=a_{l, 2 i-1}+\mathbf{j} a_{l, 2 i}, \quad 1 \leq i \leq k+1
$$

and for each $l^{\prime}\left(0 \leq l^{\prime} \leq L_{0}-1\right)$, let

$$
x_{k+1+i}=a_{l^{\prime}, 2 i-1}^{\prime}+\mathbf{j} a_{l^{\prime}, 2 i}^{\prime}, \quad 1 \leq i \leq k+1
$$

where $S^{\prime}=\left\{\left[a_{l^{\prime}, 1}^{\prime} a_{l^{\prime}, 2}^{\prime} \cdots a_{l^{\prime}, 2(k+1)}^{\prime}\right]^{T} \in \mathrm{R}^{2(k+1)}: 0 \leq l^{\prime} \leq\right.$ $\left.L_{0}-1\right\}$ is a rotated version of $S$, which is specified as: $a_{l, 2 i-1}^{\prime}+$ $\mathbf{j} a_{l, 2 i}^{\prime}=e^{\mathbf{j} \phi}\left(a_{l, 2 i-1}+\mathbf{j} a_{l, 2 i}\right)$ or equivalently

$$
\left[\begin{array}{c}
a_{l, 2 i-1}^{\prime} \\
a_{l, 2 i}^{\prime}
\end{array}\right]=\left[\begin{array}{cc}
\cos \phi & -\sin \phi \\
\sin \phi & \cos \phi
\end{array}\right]\left[\begin{array}{c}
a_{l, 2 i-1} \\
a_{l, 2 i}
\end{array}\right]
$$

for $1 \leq i \leq k+1$ and $0 \leq l \leq L_{0}-1$. The rotation angle $\phi$ will be specified later, and it depends on the sphere packing $S$. If we define

$$
C_{l, l^{\prime}}=\sqrt{\frac{2^{k} L_{0}}{E}} Q_{2^{k+1}}\left(x_{1}, x_{2}, \ldots, x_{2(k+1)}\right)
$$

then $\left\{C_{l, l^{\prime}}: 0 \leq l, l^{\prime} \leq L_{0}-1\right\}$ is a STBC with $L=L_{0}^{2}$ codewords for $M=2^{k+1}$ transmit antennas. The factor $\sqrt{2^{k} L_{0} / E}$ ensures that the resulting STBC satisfies the energy constraint. The diversity product can be calculated as [14]

$$
\begin{aligned}
\zeta= & \frac{1}{2} \sqrt{\frac{L_{0}}{2 E}} \min _{X \neq \tilde{X}}\left(\sum_{i=1}^{k+1}\left|\left(x_{i}-\tilde{x}_{i}\right)+\left(x_{i+k+1}-\tilde{x}_{i+k+1}\right)\right|^{2}\right)^{\frac{1}{4}} \\
& \times\left(\sum_{i=1}^{k+1}\left|\left(x_{i}-\tilde{x}_{i}\right)-\left(x_{i+k+1}-\tilde{x}_{i+k+1}\right)\right|^{2}\right)^{\frac{1}{4}}
\end{aligned}
$$

$$
Q_{2^{k+1}}\left(x_{1}, x_{2}, \ldots, x_{2(k+1)}\right)=\left[\begin{array}{cc}
G_{2^{k}}\left(x_{1}, x_{2}, \ldots, x_{k+1}\right) & G_{2^{k}}\left(x_{k+2}, x_{k+3}, \ldots, x_{2(k+1)}\right) \\
G_{2^{k}}\left(x_{k+2}, x_{k+3}, \ldots, x_{2(k+1)}\right) & G_{2^{k}}\left(x_{1}, x_{2}, \ldots, x_{k+1}\right)
\end{array}\right] .
$$




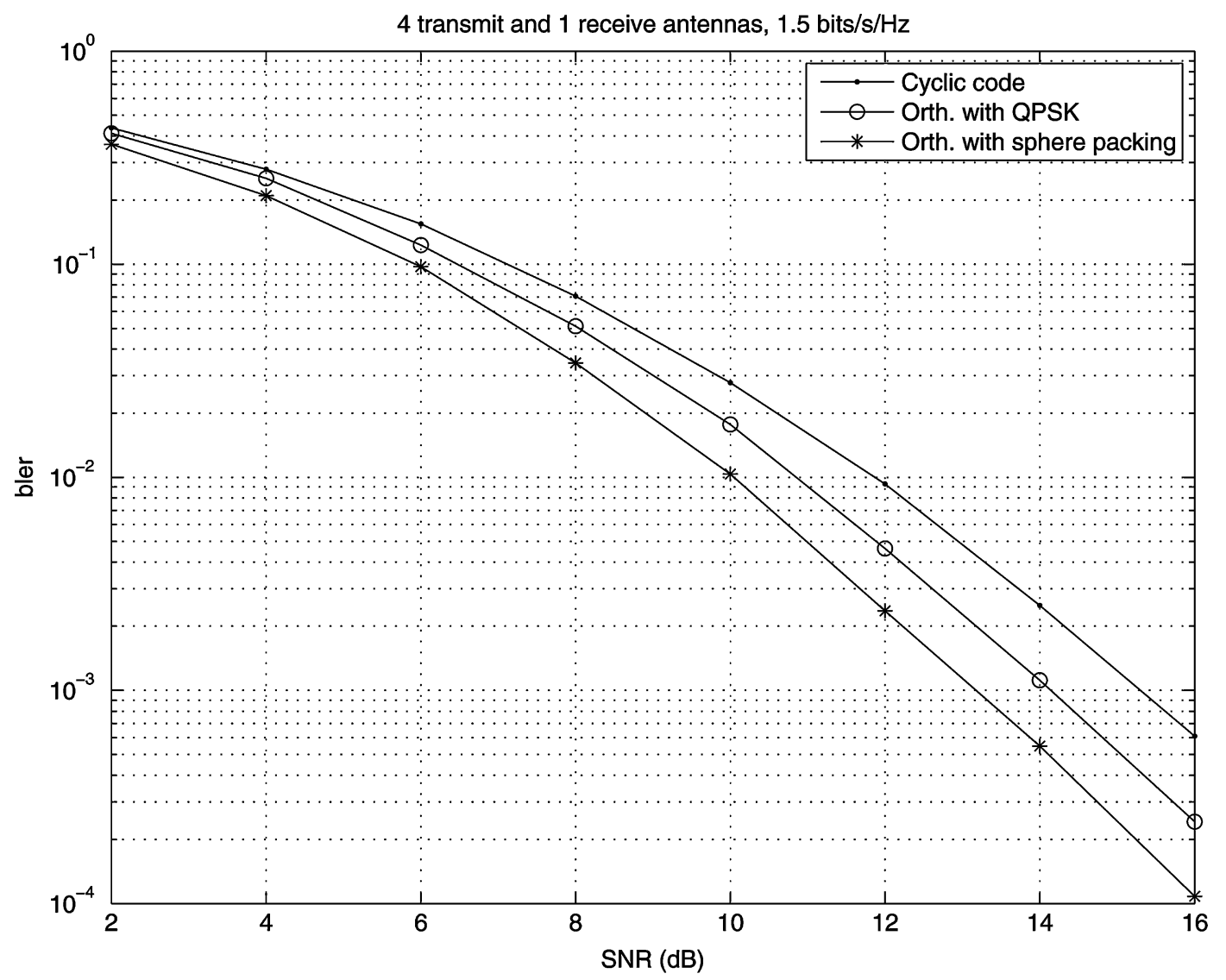

Fig. 3. Block error rate performance of cyclic codes “.," orth. with QPSK “o," and orth. with sphere packing “*.” $L=64$.

where $X=\left(x_{1}, x_{2}, \ldots, x_{2(k+1)}\right)$ and $\tilde{X}=$ $\left(\tilde{x}_{1}, \tilde{x}_{2}, \ldots, \tilde{x}_{2(k+1)}\right)$. Obviously, we have

$$
\begin{aligned}
\zeta & \leq \frac{1}{2} \sqrt{\frac{L_{0}}{2 E}} \min _{X_{1} \neq \tilde{X}_{1}}\left(\sum_{i=1}^{k+1}\left|x_{i}-\tilde{x}_{i}\right|^{2}\right)^{1 / 2} \\
& =\frac{1}{2} \sqrt{\frac{L_{0}}{2 E}} \min _{0 \leq l \neq l^{\prime} \leq L_{0}-1}\left(\sum_{i=1}^{2(k+1)}\left|a_{l, i}-a_{l^{\prime}, i}\right|^{2}\right)^{1 / 2} \\
& =\frac{1}{2} \sqrt{\frac{L_{0}}{2 E}} d_{\min }(S)
\end{aligned}
$$

where $X_{1}=\left(x_{1}, x_{2}, \ldots, x_{k+1}\right)$ and $\tilde{X}_{1}=\left(\tilde{x}_{1}, \tilde{x}_{2}, \ldots, \tilde{x}_{k+1}\right)$, and $d_{\min }(S)$ denotes the minimum Euclidean distance of the sphere packing $S$. We observe that the diversity product is upper bounded by the normalized minimum Euclidean distance of the sphere packing $S$.

For a fixed sphere packing $S$, our objective is to find an optimal rotation angle $\phi$ to maximize the diversity product $\zeta$ in (21). From the result in the previous subsection, we know that in case of the sphere packing from the four-dimensional lattice $D_{4}, \phi=\pi / 4$ is an optimal rotation angle. The following theorem generalizes this result by showing that for any sphere packing from the $2(k+1)$-dimensional lattice $D_{2(k+1)}$, which is defined as ([29, p. 117])

$$
\begin{aligned}
D_{2(k+1)} \triangleq\left\{\left[n_{1} n_{2} \ldots n_{2(k+1)}\right] \in \mathbf{Z}^{2(k+1)}\right. & : n_{1}+n_{2}+\cdots \\
& \left.+n_{2(k+1)} \text { is even }\right\}
\end{aligned}
$$

the rotation angle $\phi=\pi / 4$ is optimal in the sense that the upper bound in (22) is achieved.

Theorem 1: For any sphere packing $S$ from the $2(k+1)$-dimensional lattice $D_{2(k+1)}$, if the rotation angle in (19) is chosen as $\phi=\pi / 4$, the diversity product of the STBC defined in (20) by combining the sphere packing $S$ and the block-orthogonal design $Q_{2^{k+1}}$ is

$$
\zeta=\frac{1}{2} \sqrt{\frac{L_{0}}{2 E}} d_{\min }(S) .
$$

A proof of the theorem can be found in Appendix I. Note that the minimum Euclidean distance of the sphere packing $D_{2(k+1)}$ is $\sqrt{2}\left(\left[29\right.\right.$, p. 117]), i.e., $d_{\min }(S)=\sqrt{2}$, thus the diversity product is $\zeta=\frac{1}{2} \sqrt{\frac{L_{0}}{E}}$. From (22) and (23), we observe that in case of using the sphere packing $D_{2(k+1)}, \phi=\pi / 4$ is an optimal rotation angle. For $M=4$ transmit antennas $(k=1)$, we showed this in the previous subsection using the results of [19] which was obtained via analytical tools from algebraic number theory. Theorem 1 provides an alternative way to determine the optimal rotation angle.

\section{Simulation Results}

We present some simulation results in this section. All of the simulated communication systems had one receive antenna. Assuming that there are $L$ codewords in a STBC, and each codeword is transmitted over $T=M$ channel uses, the rate of the STBC is $\left(\log _{2} L\right) / M$ bits per channel use, which corresponds to 


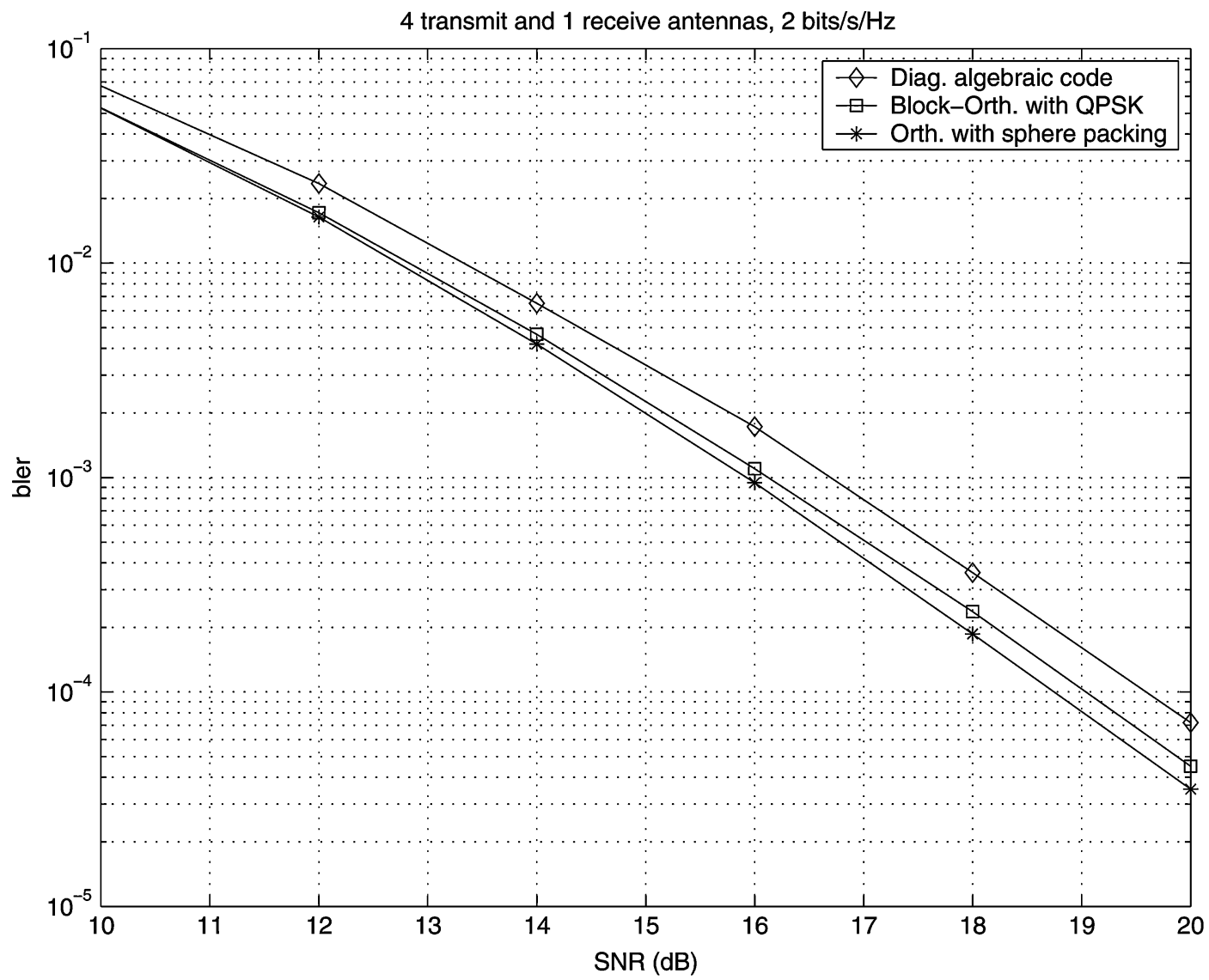

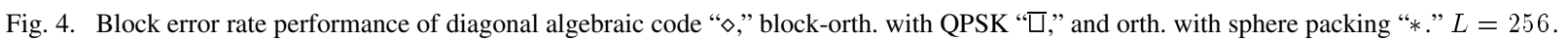

$\left(\log _{2} L\right) / M$ bits/s/Hz spectral efficiency. We assumed that the channel state information is known exactly at the receiver and the ML decoding method was used. We present block error rate (bler) versus average SNR curves.

\section{A. Two Transmit Antennas}

First, we compare the proposed scheme, orthogonal design with sphere packing $D_{4}$, with other three schemes: the conventional orthogonal design, the parametric code [17], and the cyclic code [15]. Fig. 1 shows the simulation results for $L=64$ case, resulting in a spectral efficiency of $3 \mathrm{bits} / \mathrm{s} / \mathrm{Hz}$. In order to maintain the same spectral efficiency, we simulated the conventional orthogonal design with 8 PSK and 8 QAM modulations, respectively. The curves demonstrate that the proposed method has the best performance. For example, at a bler of $10^{-2}$, the orthogonal design with sphere packing has an improvement of about $0.5 \mathrm{~dB}$ over the conventional orthogonal design with 8 QAM, $1.5 \mathrm{~dB}$ over the orthogonal design with $8 \mathrm{PSK}, 2 \mathrm{~dB}$ over the parametric code, and $4 \mathrm{~dB}$ over the cyclic code. We observe that all the bler curves have approximately the same asymptotic slope, suggesting that all these schemes achieve the same diversity order.

Fig. 2 depicts the simulation results for the $L=256$ case (spectral efficiency of $4 \mathrm{bits} / \mathrm{s} / \mathrm{Hz}$ ). Our scheme is compared with the diagonal algebraic code [21], the high rate code $B_{2}(\phi, \theta)$ [20], the Golden code [22], and the conventional orthogonal design with 16-QAM. We chose 16-QAM constellation for the diagonal algebraic code, and the corresponding unitary rotation matrix was $\frac{1}{\sqrt{2}}\left[\begin{array}{cc}1 & e^{\mathbf{j} \pi / 4} \\ 1 & -e^{\mathbf{j} \pi / 4}\end{array}\right]$. We used QPSK constellation for the high rate code $B_{2}(\phi, \theta)$ with the optimum parameters $\phi=e^{\mathbf{j} \pi / 4}$ and $\theta=e^{2 \mathbf{j} \pi / 6}$ (see [20, Sec. II-D]). The figure shows that the orthogonal design with sphere packing has an improvement of about $0.75 \mathrm{~dB}$ over the conventional orthogonal design, $1.5 \mathrm{~dB}$ over the Golden code, $2 \mathrm{~dB}$ over the diagonal algebraic code, and about $2.5 \mathrm{~dB}$ over the high rate code $B_{2}(\phi, \theta)$ at a bler of $10^{-2}$.

We also observe that in the $L=64$ case, even though the diversity product of the parametric code $(\zeta=0.3070)$ is larger than that of the orthogonal design with 8 PSK $(\zeta=0.2706)$, the simulated bler curves of Fig. 1 show that the the latter outperforms the former. A similar phenomenon can be seen in Fig. 2 for $L=256$ : the orthogonal design with 16-QAM $(\zeta=0.2237)$ has a better performance than the diagonal algebraic code $(\zeta=$ $0.2237)$, the high rate code $B_{2}(\phi, \theta)(\zeta=0.2544)$, and the Golden code $(\zeta=0.3344)$. The reason for this is that the diversity product can only characterize the worst-case pair-wise error probability, while the actual performance is governed by the whole spectrum of the determinants of the code difference matrices $C-\tilde{C}$ [27].

\section{B. Four Transmit Antennas}

For four transmit antennas $(M=4)$, we compare both orthogonal designs with sphere packing and block-orthogonal design with sphere packing to some existing approaches. The performance of the STBCs designed for $L=64$ (spectral efficiency of $1.5 \mathrm{~b} / \mathrm{s} / \mathrm{Hz}$ ) is shown in Fig. 3. The figure depicts 


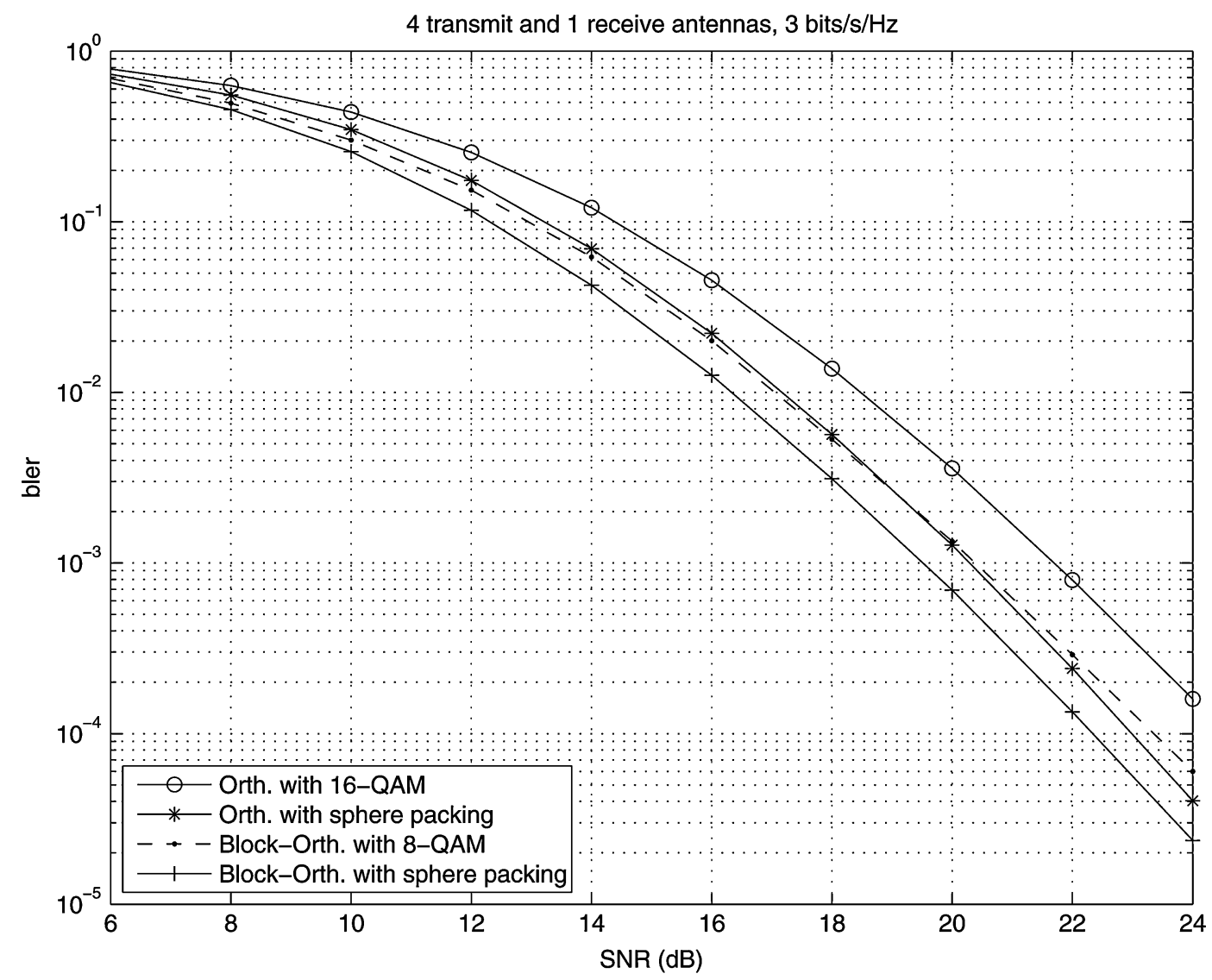

Fig. 5. Block error rate performance of orth. with 16-QAM “o," orth. with sphere packing “*," block-orth. with 8 QAM “., and block-orth. with sphere packing “+." $L=4096$.

the bler curves of the orthogonal design with sphere packing, the conventional orthogonal design with QPSK, and the cyclic code. The curves show that the proposed scheme outperforms the other two schemes. For example, at a bler of $10^{-3}$, we observe a performance improvement of about $1 \mathrm{~dB}$ compared to the conventional orthogonal design with QPSK, and about $2.5 \mathrm{~dB}$ compared to the cyclic code.

Fig. 4 contains the simulation results for the $L=256$ case (spectral efficiency of $2 \mathrm{bits} / \mathrm{s} / \mathrm{Hz}$ ). Our scheme is compared with the diagonal algebraic code [21], and the full-diversity block-orthogonal design [13], [14]. We used QPSK constellation for the diagonal algebraic code, and the corresponding unitary rotation matrix was

$$
\frac{1}{2}\left[\begin{array}{cccc}
1 & \theta & \theta^{2} & \theta^{3} \\
1 & -\theta & \theta^{2} & -\theta^{3} \\
1 & \mathbf{j} \theta & -\theta^{2} & -\mathbf{j} \theta^{3} \\
1 & -\mathbf{j} \theta & -\theta^{2} & \mathbf{j} \theta^{3}
\end{array}\right], \quad \theta=e^{\mathbf{j} \pi / 8} .
$$

We also chose QPSK constellation for the full-diversity blockorthogonal design to maintain the same spectral efficiency. We can see that at a bler of $10^{-4}$, the orthogonal design with sphere packing outperforms the block-orthogonal design about $0.5 \mathrm{~dB}$, and outperforms the diagonal algebraic code about $1.75 \mathrm{~dB}$.

Finally, in Fig. 5, we show that the performance can be further improved if we construct STBCs from block-orthogonal designs with sphere packing. Fig. 5 provides the simulation results for the $L=4096$ case, giving a spectral efficiency of $3 \mathrm{bits} / \mathrm{s} / \mathrm{Hz}$. We compared four schemes: the conventional orthogonal design with 16-QAM, the orthogonal design with sphere packing, the block-orthogonal design with 8-QAM, and the block-orthogonal design with sphere packing. We constructed the STBC from orthogonal design with sphere packing by taking $L=$ 4096 points from $E_{6}$, and the STBC from block-orthogonal design with sphere packing by taking $L_{0}=64$ points from $D_{4}$. From the simulation results we observe that at a bler of $10^{-4}$, the orthogonal design with sphere packing is better than the conventional orthogonal design about $1.5 \mathrm{~dB}$, and the block-orthogonal design with sphere packing has an improvement of about $0.75 \mathrm{~dB}$ over orthogonal design with sphere packing, and $1 \mathrm{~dB}$ over the block-orthogonal design with 8-QAM. Moreover, we can see that the performance curve of the block-orthogonal design with sphere packing has almost the same asymptotic slope as the other two schemes. This confirms that the STBC constructed from the block-orthogonal design with the sphere packing $D_{4}$ (with $\phi=\pi / 4$ ) achieves full diversity.

\section{CONCLUSION}

In this paper, we focused on the problem of designing STBCs from orthogonal and block-orthogonal designs. We proposed a code construction method by combining orthogonal and block-orthogonal designs with sphere packing. We constructed codes for two and four transmit antennas and 1.5, 2, 3, and 4 bits $/ \mathrm{s} / \mathrm{Hz}$ spectral efficiencies. Both the theoretical diversity product values and the simulation results demonstrate the superior performance of the proposed method. In case of two transmit and one receive antennas and a spectral efficiency of $4 \mathrm{bits} / \mathrm{s} / \mathrm{Hz}$, we observed a coding gain of about $0.75 \mathrm{~dB}$ over 
the conventional orthogonal design, $1.5 \mathrm{~dB}$ over the Golden code, $2 \mathrm{~dB}$ over the diagonal algebraic code, and about $2.5 \mathrm{~dB}$ over the high rate code $B_{2}(\phi, \theta)$ at a bler of $10^{-2}$. By jointly designing the symbols in the orthogonal and block-orthogonal designs, we exploited the additional degrees of freedom to further improve the performance at the expense of having to decode the symbols jointly. However, the decoding complexity can be significantly reduced if sphere decoding algorithms [25], [26] are used in which searching radius can be adjusted to reduce the decoding complexity and there is tradeoff between the decoding complexity and performance degradation. Finally, we would like to mention that the code designs discussed in this paper were optimized based on pairwise bler performance. It would be interesting to optimize the code designs based on bit-error-rate performance and investigate optimal bit labeling in future work.

\section{APPENDIX}

\section{PROOF OF THEOREM 1}

Since the diversity product is upper bounded by $\frac{1}{2} \sqrt{\frac{L_{0}}{2 E}} d_{\min }(S)$, and $d_{\min }(S)=\sqrt{2}$ in this case, in order to prove the theorem, it is sufficient to show that

$$
\zeta \geq \frac{1}{2} \sqrt{\frac{L_{0}}{E}} .
$$

By Cauchy's inequality, the diversity product in (21) can be lower bounded as follows:

$\zeta \geq \frac{1}{2} \sqrt{\frac{L_{0}}{2 E}} \min _{X \neq \tilde{X}}$

$$
\times\left(\sum_{i=1}^{k+1}\left|\left(x_{i}-\tilde{x}_{i}\right)^{2}-\left(x_{i+k+1}-\tilde{x}_{i+k+1}\right)^{2}\right|\right)^{1 / 2}
$$

where $X=\left(x_{1}, x_{2}, \ldots, x_{2(k+1)}\right)$ and $\tilde{X}$ $\left(\tilde{x}_{1}, \tilde{x}_{2}, \ldots, \tilde{x}_{2(k+1)}\right)$. Defining $d$ as

$$
d \triangleq\left(\sum_{i=1}^{k+1}\left|\left(x_{i}-\tilde{x}_{i}\right)^{2}-\left(x_{i+k+1}-\tilde{x}_{i+k+1}\right)^{2}\right|\right)^{1 / 2}
$$

in order to show $\zeta \geq \frac{1}{2} \sqrt{\frac{L_{0}}{E}}$, it is sufficient to prove that $d \geq \sqrt{2}$ for any $X \neq \tilde{X}$.

For any pair of vectors $X_{1}=\left(x_{1}, x_{2}, \ldots, x_{k+1}\right)$ and $\tilde{X}_{1}=\left(\tilde{x}_{1}, \tilde{x}_{2}, \ldots, \tilde{x}_{k+1}\right)$ from the $2(k+1)$-dimensional lattice $D_{2(k+1)}$, the difference vector $X_{1}-\tilde{X}_{1}$ can be represented as

$$
X_{1}-\tilde{X}_{1}=\left(n_{1}+\mathbf{j} n_{2}, n_{3}+\mathbf{j} n_{4}, \ldots, n_{2 k+1}+\mathbf{j} n_{2 k+2}\right)
$$

for some integers $n_{1}, n_{2}, \ldots, n_{2(k+1)}$. According to the definition of the lattice $D_{2(k+1)}$, we know that $n_{1}+$ $n_{2}+\cdots+n_{2(k+1)}$ is even. Similarly, for any pair of vectors $X_{2}=\left(x_{k+2}, x_{k+3}, \ldots, x_{2(k+1)}\right)$ and $\tilde{X}_{2}=$ $\left(\tilde{x}_{k+2}, \tilde{x}_{k+3}, \ldots, \tilde{x}_{2(k+1)}\right)$ from the rotated version of the lattice $D_{2(k+1)}$ with a rotation angle $\phi=\pi / 4$ as defined in (19), the difference vector $X_{2}-\tilde{X}_{2}$ can be represented as

$$
\begin{aligned}
X_{2} & -\tilde{X}_{2} \\
& =e^{\mathbf{j} \pi / 4}\left(m_{1}+\mathbf{j} m_{2}, m_{3}+\mathbf{j} m_{4}, \ldots, m_{2 k+1}+\mathbf{j} m_{2 k+2}\right)
\end{aligned}
$$

for some integers $m_{1}, m_{2}, \ldots, m_{2(k+1)}$. The sum of these integers $m_{1}, m_{2}, \ldots, m_{2(k+1)}$ is also even. Substituting (27) and (28) into (26), we have

$$
\begin{aligned}
d^{2}= & \sum_{i=1}^{k+1}\left|\left(n_{2 i-1}+\mathbf{j} n_{2 i}\right)^{2}-e^{\mathbf{j} \pi / 2}\left(m_{2 i-1}+\mathbf{j} m_{2 i}\right)^{2}\right| \\
= & \sum_{i=1}^{k+1}\left[\left(n_{2 i-1}^{2}-n_{2 i}^{2}+2 m_{2 i-1} m_{2 i}\right)^{2}\right. \\
& \left.+\left(m_{2 i}^{2}-m_{2 i-1}^{2}+2 n_{2 i-1} n_{2 i}\right)^{2}\right]^{1 / 2} \triangleq \sum_{i=1}^{k+1} A_{i} .
\end{aligned}
$$

Since all of $n_{1}, n_{2}, \ldots, n_{2(k+1)}$ and $m_{1}, m_{2}, \ldots, m_{2(k+1)}$ are integers, so if there are two indices $i_{1}$ and $i_{2}\left(i_{1} \neq i_{2}\right)$ such that $A_{i_{1}} \neq 0$ and $A_{i_{2}} \neq 0$, then $d^{2} \geq A_{i_{1}}+A_{i_{2}} \geq 2$, which is the desired result.

In the following, we will prove that it is impossible to have the case that all of $A_{i}(1 \leq i \leq k+1)$ are zeros under the constraint that $X \neq \tilde{X}$, i.e., $n_{1}, n_{2}, \ldots, n_{2(k+1)}$ and $m_{1}, m_{2}, \ldots, m_{2(k+1)}$ cannot be zero at the same time. Finally, if there is only one nonzero $A_{i_{0}}$, i.e., $A_{i_{0}} \neq 0$ and $A_{i}=0$ for all $i \neq i_{0}$, we will show that $A_{i_{0}} \geq 2$, which also implies that $d^{2} \geq 2$. The rest of proof is divided into three steps.

Step 1: First, we show that for any $i, 1 \leq i \leq k+1$, if $A_{i}=0$, then $n_{2 i-1}=n_{2 i}=0$ and $m_{2 i-1}=m_{2 i}=0$.

Without loss of generality, we assume that $A_{1}=0$. The proof for $A_{i}=0(2 \leq i \leq k+1)$ is similar. With the assumption that $A_{1}=0$, we know that both $n_{1}^{2}-n_{2}^{2}+2 m_{1} m_{2}$ and $m_{2}^{2}-m_{1}^{2}+$ $2 n_{1} n_{2}$ are zeros, i.e.

$$
\begin{aligned}
n_{2}^{2}-n_{1}^{2} & =2 m_{1} m_{2} \\
m_{1}^{2}-m_{2}^{2} & =2 n_{1} n_{2} .
\end{aligned}
$$

The equation in (29) implies that either both $n_{1}$ and $n_{2}$ are odd, or both $n_{1}$ and $n_{2}$ are even. If both $n_{1}$ and $n_{2}$ are odd, denoted as $n_{1}=2 \tilde{n}_{1}+1$ and $n_{2}=2 \tilde{n}_{2}+1$, then substituting them into (29), we have $4\left(\tilde{n}_{2}^{2}+\tilde{n}_{2}-\tilde{n}_{1}^{2}-\tilde{n}_{1}\right)=2 m_{1} m_{2}$. It follows that one of $m_{1}$ and $m_{2}$ is even. On the other hand, from (30) we know that either both $m_{1}$ and $m_{2}$ are odd, or both $m_{1}$ and $m_{2}$ are even. Thus we conclude that both $m_{1}$ and $m_{2}$ are even, denoted as $m_{1}=2 \tilde{m}_{1}$ and $m_{2}=2 \tilde{m}_{2}$. Substituting them into (30), we have $4\left(\tilde{m}_{1}^{2}-\tilde{m}_{2}^{2}\right)=2 n_{1} n_{2}$, which is contradictory to the assumption that both $n_{1}$ and $n_{2}$ are odd. Thus, both $n_{1}$ and $n_{2}$ must be even, denoted as $n_{1}=2 \tilde{n}_{1}$ and $n_{2}=2 \tilde{n}_{2}$.

Similarly, we can prove that both $m_{1}$ and $m_{2}$ must be even, denoted as $m_{1}=2 \tilde{m}_{1}$ and $m_{2}=2 \tilde{m}_{2}$. Substituting $n_{1}=$ $2 \tilde{n}_{1}, n_{2}=2 \tilde{n}_{2}, m_{1}=2 \tilde{m}_{1}$ and $m_{2}=2 \tilde{m}_{2}$ into (29) and (30), we obtain

$$
\begin{aligned}
\tilde{n}_{2}^{2}-\tilde{n}_{1}^{2} & =2 \tilde{m}_{1} \tilde{m}_{2} \\
\tilde{m}_{1}^{2}-\tilde{m}_{2}^{2} & =2 \tilde{n}_{1} \tilde{n}_{2} .
\end{aligned}
$$

Repeating the above argument, we can prove that all of $\tilde{n}_{1}, \tilde{n}_{2}, \tilde{m}_{1}$ and $\tilde{m}_{2}$ are even. We can continue this process repetitively. Since all of $n_{1}, n_{2}, m_{1}$ and $m_{2}$ are finite integers, we conclude that some of $n_{1}, n_{2}, m_{1}$ and $m_{2}$ must be zero.

If $n_{1}=0$, from (30) we have $m_{1}^{2}=m_{2}^{2}$, i.e., $m_{1}= \pm m_{2}$. Substituting $n_{1}=0$ and $m_{1}= \pm m_{2}$ into (29), we arrive at 
$n_{1}=2 m_{1} m_{2}= \pm 2 m_{2}^{2}$. It follows that $n_{2}=0$ and $m_{2}=0$, since both $n_{2}$ and $m_{2}$ are integers. Thus, all of $n_{1}, n_{2}, m_{1}$ and $m_{2}$ are zeros. If $n_{2}=0$ or $m_{1}=0$ or $m_{2}=0$, we can prove that all of $n_{1}, n_{2}, m_{1}$ and $m_{2}$ are zeros in a similar way.

Step 2: In this step, we show that under the constraint that $X \neq \tilde{X}$, one of $A_{i}(1 \leq i \leq k+1)$ must be nonzero. According to the result in Step 1, we know that for any $i, 1 \leq i \leq k+1$, if $A_{i}=0$, then $n_{2 i-1}=n_{2 i}=0$ and $m_{2 i-1}=m_{2 i}=0$. If all of $A_{i}(1 \leq i \leq k+1)$ are zeros, then all of $n_{1}, n_{2}, \ldots, n_{2(k+1)}$ and $m_{1}, m_{2}, \ldots, m_{2(k+1)}$ are zeros, which is contradictory to the constraint that $X \neq X$. As a result, for two distinct vectors $X$ and $\tilde{X}$, at least one of the $A_{i}$ 's must be nonzero. Therefore, the only remaining case we have to consider is when exactly one $A_{i}$ is nonzero.

Step 3: Finally, we show that if there is one index $i_{0}$ such that $A_{i_{0}} \neq 0$ and $A_{i}=0$ for all $i \neq i_{0}$, then $A_{i_{0}} \geq 2$. Without loss of generality, we assume that $A_{1} \neq 0$ and $A_{i}=0$ for all $2 \leq i \leq k+1$. From the result of Step 1, we know that $n_{2 i-1}=n_{2 i}=0$ and $m_{2 i-1}=m_{2 i}=0$ for all $2 \leq i \leq k+$ 1. Since all of $n_{1}, n_{2}, \ldots, n_{2(k+1)}$ and $m_{1}, m_{2}, \ldots, m_{2(k+1)}$ cannot be zero at the same time, one of $n_{1}, n_{2}, m_{1}, m_{2}$ must be nonzero. Moreover, since both $n_{1}+n_{2}+\cdots+n_{2(k+1)}$ and $m_{1}+m_{2}+\cdots+m_{2(k+1)}$ are even, both $n_{1}+n_{2}$ and $m_{1}+m_{2}$ are even in this case.

From the assumption that $A_{1} \neq 0$, i.e., $A_{1}=\left[\left(n_{1}^{2}-n_{2}^{2}+\right.\right.$ $\left.\left.2 m_{1} m_{2}\right)^{2}+\left(m_{2}^{2}-m_{1}^{2}+2 n_{1} n_{2}\right)^{2}\right]^{1 / 2} \neq 0$, we know that at least one of $n_{1}^{2}-n_{2}^{2}+2 m_{1} m_{2}$ and $m_{2}^{2}-m_{1}^{2}+2 n_{1} n_{2}$ must be nonzero. Since

$$
\begin{aligned}
& n_{1}^{2}-n_{2}^{2}+2 m_{1} m_{2}=\left(n_{1}+n_{2}\right)\left(n_{1}-n_{2}\right)+2 m_{1} m_{2} \\
& m_{2}^{2}-m_{1}^{2}+2 n_{1} n_{2}=\left(m_{2}+m_{1}\right)\left(m_{2}-m_{1}\right)+2 n_{1} n_{2}
\end{aligned}
$$

and both $n_{1}+n_{2}$ and $m_{1}+m_{2}$ are even, both $n_{1}^{2}-n_{2}^{2}+2 m_{1} m_{2}$ and $m_{2}^{2}-m_{1}^{2}+2 n_{1} n_{2}$ are even. If $n_{1}^{2}-n_{2}^{2}+2 m_{1} m_{2} \neq 0$, then $\left|n_{1}^{2}-n_{2}^{2}+2 m_{1} m_{2}\right| \geq 2$. If $m_{2}^{2}-m_{1}^{2}+2 n_{1} n_{2} \neq 0$, then $\left|m_{2}^{2}-m_{1}^{2}+2 n_{1} n_{2}\right| \geq 2$. Therefore, we conclude that $A_{1} \geq 2$. Thus, we have proved the theorem completely.

\section{REFERENCES}

[1] J.-C. Guey, M. P. Fitz, M. R. Bell, and W.-Y. Kuo, "Signal design for transmitter diversity wireless communication systems over rayleigh fading channels," in Proc. IEEE VTC'96, pp. 136-140.

[2] V. Tarokh, N. Seshadri, and A. R. Calderbank, "Space-time codes for high data rate wireless communication: Performance criterion and code construction," IEEE Inf. Theory, vol. 44, no. 2, pp. 744-765, 1998.

[3] S. Alamouti, "A simple transmit diversity technique for wireless communications," IEEE J. Sel. Areas Commun., vol. 16, no. 8, pp. 1451-1458, 1998.

[4] V. Tarokh, H. Jafarkhani, and A. R. Calderbank, "Space-time block codes from orthogonal designs," IEEE Trans. Inf. Theory, vol. 45, no. 5, pp. 1456-1467, 1999.

[5] G. Ganesan and P. Stoica, "Space-time block codes: A maximum SNR approach," IEEE Trans. Inf. Theory, vol. 47, pp. 1650-1656, May 2001.

[6] O. Tirkkonen and A. Hottinen, "Square-matrix embeddable space-time block codes for complex signal constellations," IEEE Trans. Inf. Theory, vol. 48, no. 2, pp. 384-395, Feb. 2002.

[7] W. Su and X.-G. Xia, "On space-time block codes from complex orthogonal designs," Wireless Pers. Commun., vol. 25, no. 1, pp. 1-26, Apr. 2003.
[8] W. Su, X.-G. Xia, and K. J. R. Liu, "A systematic design of high-rate complex orthogonal space-time block codes," IEEE Commun. Lett., vol. 8, no. 6, pp. 380-382, Jun. 2004.

[9] H. Jafarkhani, "A quasi-orthogonal space-time block code," IEEE Trans. Commun., vol. 49, no. 1, pp. 1-4, 2001.

[10] O. Tirkkonen, A. Boariu, and A. Hottinen, "Minimal non-orthogonality rate 1 space-time block code for 3+ Tx antennas," in Proc. IEEE 6th Int. Symp. Spread-Spectrum Tech. \& Appl. (ISSSTA 2000), Sep. 2000, pp. $429-432$.

[11] A. Hottinen and O. Tirkkonen, "Precoder designs for high-rate spacetime block codes," in Proc. CISS, Princeton, NJ, Mar. 2004.

[12] N. Sharma and C. B. Papadias, "Improved quasi-orthogonal codes through constellation rotation,” IEEE Trans. Commun., vol. 51, no. 3, pp. 332-335, Mar. 2003.

[13] W. Su and X.-G. Xia, "Quasi-orthogonal space-time block codes with full diversity," in Proc. IEEE GLOBECOM'02, 2002, vol. 2, pp. 1098-1102.

[14] W. Su and X.-G. Xia, "Signal constellations for quasi-orthogonal space-time block codes with full diversity," IEEE Trans. Inf. Theory, vol. 50, no. 10, pp. 2331-2347, Oct. 2004.

[15] B. M. Hochwald and W. Sweldens, "Differential unitary space-time modulation," IEEE Trans. Commun., vol. 48, pp. 2041-2052, Dec. 2000.

[16] A. Shokrollahi, B. Hassibi, B. M. Hochwald, and W. Sweldens, "Representation theory for high-rate multiple-antenna code design," IEEE Trans. Inf. Theory, vol. 47, pp. 2335-2367, Sept. 2001.

[17] X.-B. Liang and X.-G. Xia, "Unitary signal constellations for differential space-time modulation with two transmit antennas: Parametric codes, optimal designs and bounds," IEEE Trans. Inf. Theory, vol. 48, pp. 2291-2322, Aug. 2002.

[18] B. Hassibi and B. M. Hochwald, "Cayley differential unitary spacetime codes," IEEE Trans. Inf. Theory, vol. 48, no. 6, pp. 1485-1503, Jun. 2002.

[19] X. Giraud, E. Boutillon, and J.-C. Belfiore, "Algebraic tools to build modulation schemes for fading channels," IEEE Trans. Inf. Theory, vol. 43, pp. 938-952, May 1997.

[20] M. Damen and N. C. Beaulieu, "On two high-rate algebraic space-time codes," IEEE Trans. Inf. Theory, vol. 49, no. 4, pp. 1059-1063, Apr. 2003.

[21] Y. Xin, Z. Wang, and G. Giannakis, "Space-time diversity systems based on linear constellation precoding," IEEE Trans. Wireless Commun., vol. 2, no. 2, pp. 294-309, Mar. 2003.

[22] J.-C. Belfiore, G. Rekaya, and E. Viterbo, "The Golden code: A $2 \times 2$ full-rate space-time code with nonvanishing determinants," IEEE Trans. Inf. Theory, vol. 51, no. 4, pp. 1432-1436, Apr. 2005.

[23] F. Oggier, G. Rekaya, J.-C. Belfiore, and E. Viterbo, "Perfect space-time block codes," IEEE Trans. Inf. Theory, vol. 52, no. 9, pp. 3885-3902, Sep. 2006.

[24] A. V. Geramita and J. Seberry, Orthogonal Designs, Quadratic Forms and Hadamard Matrices, Lecture Notes in Pure and Applied Mathematics. New York: Marcel Dekker, 1979, vol. 43.

[25] H. Vikalo and B. Hassibi, "Maximum likelihood sequence detection of multiple antenna systems over dispersive channels via sphere decoding," EURASIP J. Appl. Signal Processing, vol. 2002, no. 5, pp. 525-531, 2002.

[26] Z. Safar, W. Su, and K. J. R. Liu, "A fast sphere decoding algorithm for space-frequency codes," EURASIP J. Appl. Signal Processing, vol. 2006, pp. 1-14, 2006.

[27] R. Grover, W. Su, and D. A. Pados, "An $8 \times 8$ quasi-orthogonal STBC form for transmissions over eight or four antennas," IEEE Trans. Wireless Commun., vol. 7, no. 12, pp. 4777-4785, Dec. 2008.

[28] R. A. Horn and C. R. Johnson, Matrix Analysis. Cambridge, U.K.: Cambridge Univ. Press, 1985.

[29] J. H. Conway and N. J. A. Sloane, Sphere Packings, Lattices and Groups, 2rd ed. New York: Springer-Verlag, 1999.

Weifeng Su (M'03) received the Ph.D. degree in electrical engineering from the University of Delaware, Newark, in 2002. He received the B.S. and Ph.D. degrees in applied mathematics from Nankai University, Tianjin, China, in 1994 and 1999 , respectively. 
His research interests span a broad range of areas from signal processing to wireless communications and networking, including space-time coding and modulation for MIMO wireless communications, MIMO-OFDM systems, cooperative communications for wireless networks, and ultrawideband (UWB) communications.

He has been an Assistant Professor at the Department of Electrical Engineering, State University of New York (SUNY) at Buffalo since March 2005. From June 2002 to March 2005, he was a Postdoctoral Research Associate with the Department of Electrical and Computer Engineering and the Institute for Systems Research (ISR), University of Maryland, College Park.

Dr. Su received the Signal Processing and Communications Faculty Award from the University of Delaware in 2002 as an outstanding graduate student in the field of signal processing and communications. In 2005, he received the Invention of the Year Award from the University of Maryland. He has been an Associate Editor of IEEE TRANSACTIONS ON VEHICULAR TECHNOLOGY, IEEE SignAl PROCESSING LeTTERS, and a Guest Editor of Special Issue on Cooperative Communications and Networking of IEEE JOURNAL OF SELECTED AREAS IN COMMUNICATIONS.

Zoltan Safar (M'04) received the University Diploma in electrical engineering from the Technical University of Budapest, Budapest, Hungary, in 1996 and the M.S. and Ph.D. degrees in electrical and computer engineering from the University of Maryland, College Park, in 2001 and 2003, respectively.

Currently, he is a Senior Engineer with the US R\&D Center, Samsung Electro-Mechanics America, Inc. in Atlanta, GA. From 2003 to 2005, he was an Assistant Professor in the Department of Innovation, IT University of Copenhagen, Copenhagen, Denmark. From 2005 to 2007, he was a Senior Design Engineer with Modem System Design, Nokia, Copenhagen. His research interests include wireless communications signal processing, with particular focus on wireless OFDM receiver design, MIMO communication systems, and space-time and space-frequency coding.

Dr. Safar was the recipient of the Outstanding Systems Engineering Graduate Student Award from the Institute for Systems Research, University of Maryland in 2003, and the Invention of the Year Award (together with W. Su and K. J. R. Liu) from the University of Maryland in 2004.
K. J. Ray Liu (F'03) received the B.S. degree from the National Taiwan University and the Ph.D. degree from University of California, Los Angeles, both in electrical engineering.

$\mathrm{He}$ is a Distinguished Scholar-Teacher at the University of Maryland, College Park. He is an Associate Chair of Graduate Studies and Research of the Electrical and Computer Engineering Department and leads the Maryland Signals and Information Group, conducting research encompassing broad aspects of information technology including communications and networking, information forensics and security, multimedia signal processing, and biomedical technology. His recent books include Cooperative Communications and Networking (Cambridge University Press, 2008); Resource Allocation for Wireless Networks: Basics, Techniques, and Applications (Cambridge University Press, 2008), Ultra-Wideband Communication Systems: The Multiband OFDM Approach (IEEE/Wiley, 2007), Network-Aware Security for Group Communications (Springer-Verlag, 2007), Multimedia Fingerprinting Forensics for Traitor Tracing (Hindawi, 2007), and Handbook on Array Processing and Sensor Networks (IEEE/ Wiley, 2009).

Dr. Liu is the recipient of numerous honors and awards including best paper awards from IEEE Signal Processing Society (twice), IEEE Vehicular Technology Society, and EURASIP; IEEE Signal Processing Society Distinguished Lecturer, EURASIP Meritorious Service Award, and National Science Foundation Young Investigator Award. He also received various teaching and research recognitions from the University of Maryland including university-level Invention of the Year Award, and Poole and Kent Company Senior Faculty Teaching Award as well as Outstanding Faculty Research Award, both from A. James Clark School of Engineering Faculty. He is Vice President-Publications and on the Board of Governors of the IEEE Signal Processing Society. He was the Editor-in-Chief of IEEE SIGNAL PROCESSING MAGAZINE and the founding Editor-in-Chief of EURASIP Journal on Applied Signal Processing. 